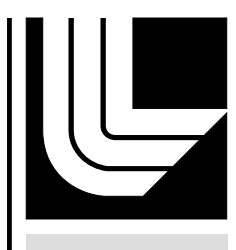

LAW RENCE LIVERMORE N A T IO N A L LABORATORY

Seeing the Universe in a Grain of Dust

A. Hazi

September 21, 2005 
This document was prepared as an account of work sponsored by an agency of the United States Government. Neither the United States Government nor the University of California nor any of their employees, makes any warranty, express or implied, or assumes any legal liability or responsibility for the accuracy, completeness, or usefulness of any information, apparatus, product, or process disclosed, or represents that its use would not infringe privately owned rights. Reference herein to any specific commercial product, process, or service by trade name, trademark, manufacturer, or otherwise, does not necessarily constitute or imply its endorsement, recommendation, or favoring by the United States Government or the University of California. The views and opinions of authors expressed herein do not necessarily state or reflect those of the United States Government or the University of California, and shall not be used for advertising or product endorsement purposes.

This work was performed under the auspices of the U.S. Department of Energy by University of California, Lawrence Livermore National Laboratory under Contract W-7405-Eng-48. 
$\because$

\section{Seeing the Universe in a Grain of Dust $\because$ $+$}

MAGINE traveling halfway to Jupiter-3.2 billion kilometers for a small handful of comet dust. That's the mission for the National Aeronautics and Space Administration's (NASA's) Stardust spacecraft launched on February 7, 1999. This past January, Stardust flew by Comet Wild 2's nucleus and through a halo of gases and dust at the comet's head, collecting cometary dust particles released from the surface just hours before. In 2006, the spacecraft will deliver the less than 1 milligram of particles to Earth. A Lawrence Livermore team is perfecting ways to extract and analyze the tiny

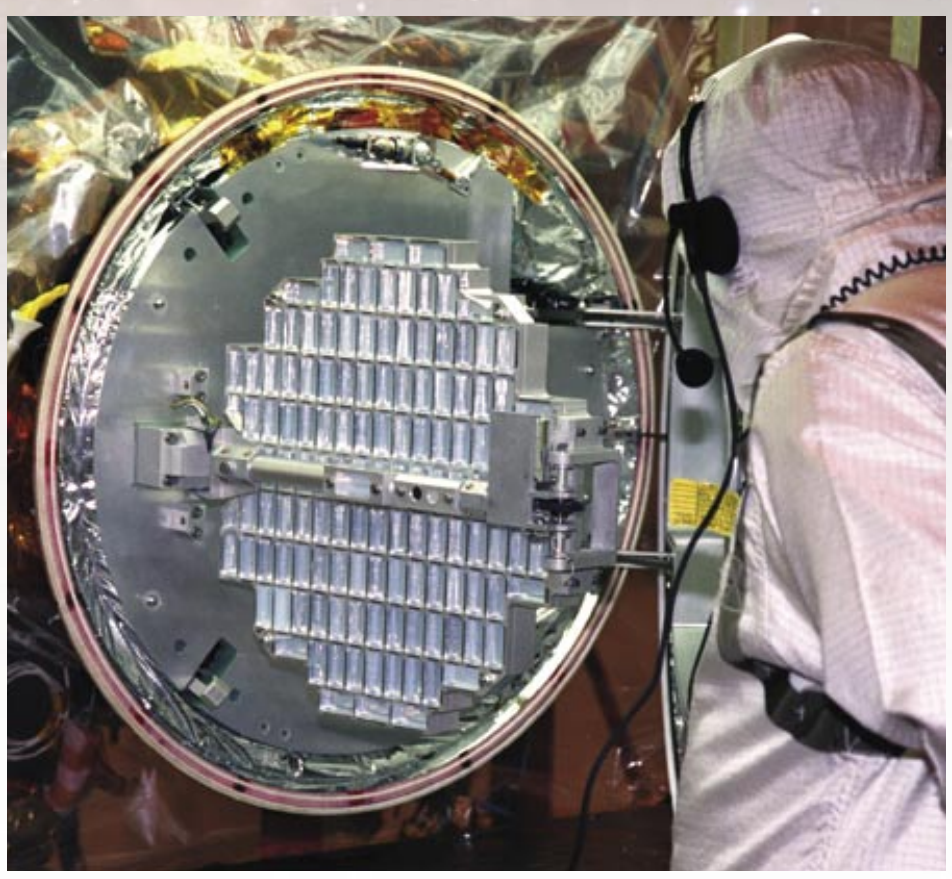

The Stardust spacecraft's collector grid, which is filled with aerogel, was designed to capture particles from Comet Wild 2 as the spacecraft flew through the comet's dust and gas cloud in January 2004. Because the aerogel is composed of 99 percent air, it can collect and store fast-moving dust particles without damaging them.

Reprinted from Science \& Technology Review, June 2004 UCRL-TR-215559 particles using its new focused-ion-beam instrument and SuperSTEM, a scanning transmission electron microscope.

Stardust is the first NASA space mission dedicated solely to collecting comet dust and will be the first to return material from a comet to Earth. Comets are the oldest and most primitive bodies in the solar system. They are formed from frozen gas, water, and interstellar dust and may have brought water to Earth, making life possible. Wild 2-pronounced "Vilt 2" after the name of its Swiss discoverer-was formed with the Sun and the rest of the solar system 4.5 billion years ago. For billions of years, it has circled the Sun in the Kuiper Belt, a region beyond the orbit of Neptune. Scientists think comets from this region have escaped the warming, vaporization, and collisions that have altered matter in the inner solar system. Unlike Halley's Comet, which has been altered as a result of orbiting the Sun for a long time, Wild 2's pristine composition is expected to offer a rich source of information about the solar system's potential building blocks.

As the 5-meter-long Stardust spacecraft traveled through Wild 2's dust and gas cloud, to within about 100 kilometers of the comet's nucleus, particles were captured in the spacecraft's collector grid. The 1,000-square-centimeter grid is filled with the silica-based material aerogel, whose lightness minimizes damage to the grains as they encounter the spacecraft at a speed of about 21,000 kilometers per hour-or six times faster than a bullet. In the late 1980s, Livermore scientists developed an aerogel made up of 99 percent air, making it ideal for NASA projects. Mission planners expect to have collected more than 1,000 grains between 2 to 5 nanometers in diameter. Most of the grains will be heterogeneous aggregates of carbonaceous matter, glass, and crystals.

Livermore is part of the Bay Area Particle Analysis Consortium (BayPac) formed to develop regional expertise on interplanetary dust particles. BayPac's members include University of California (UC) at Berkeley, UC Davis, Lawrence Berkeley National Laboratory, and Stanford University. Funding for SuperSTEMthe first of its kind in the world-comes from NASA and Livermore's Laboratory Directed Research and Development 

shows the nucleus of Comet Wild 2, taken by the Stardust spacecraft in January 2004.
This image

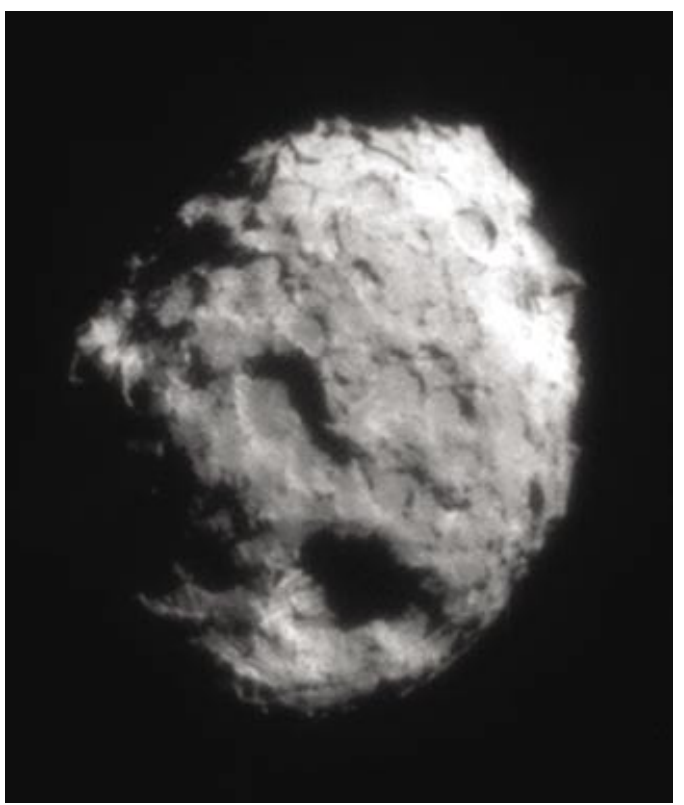

(a) Interstellar dust tracks are shown trapped in a sample of aerogel from a collector grid. (b) The keystone technique allows scientists to extract a fragmented particle from a sample of aerogel. The finergrain material distributed along the impact tracks must be recovered for comprehensive analysis of comet dust. The technique was developed at the University of California (UC) at Berkeley. (Image courtesy Space Sciences Laboratory, UC Berkeley.)
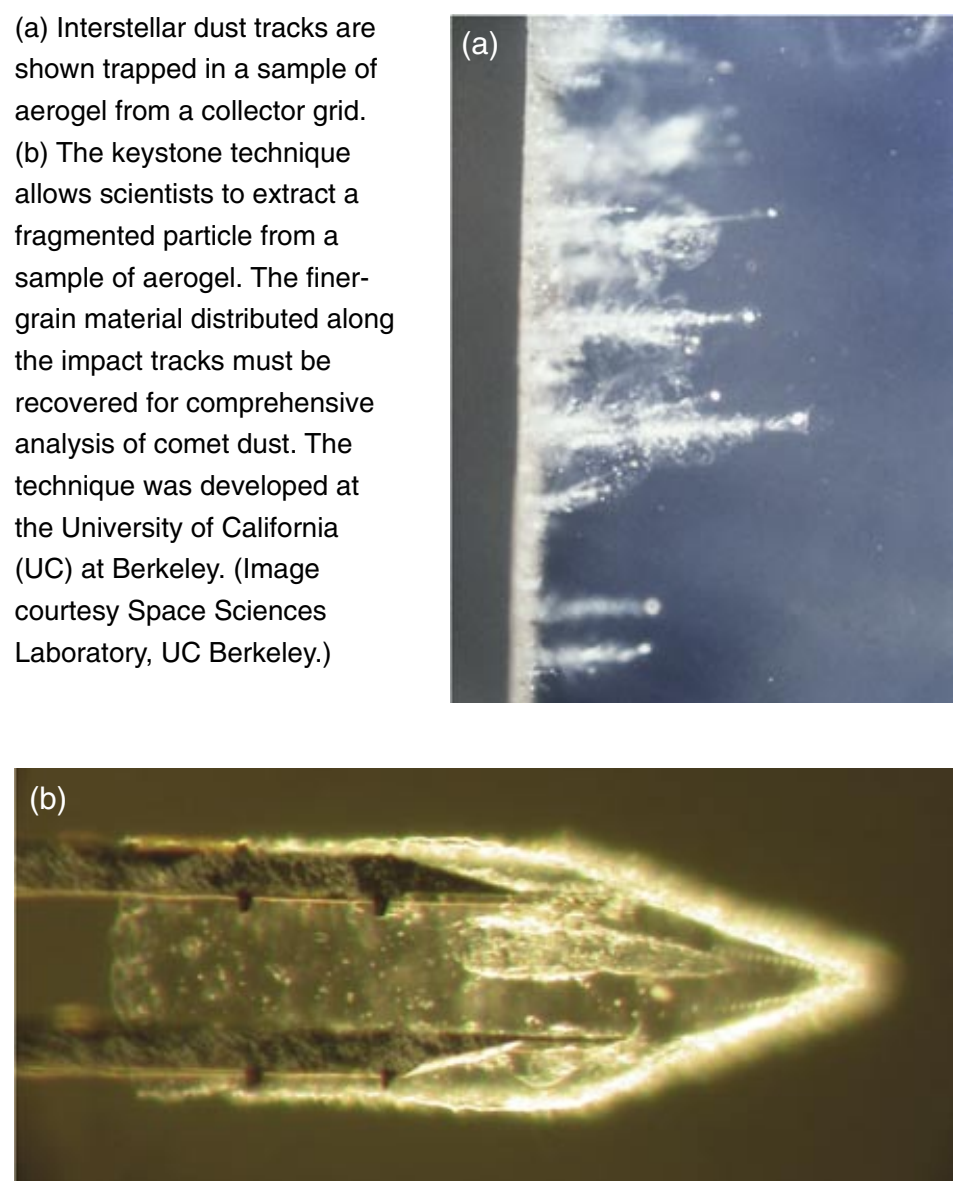

Program. John Bradley, director of the Laboratory's Institute of Geophysics and Planetary Physics, says, "This consortium provides a unique opportunity for a collaboration between universities and national laboratories in the San Francisco Bay Area to work together on a NASA mission."

\section{Perfecting Extraction and Analysis Techniques}

The particles collected by Stardust will be extremely small, so analytical instruments with a spatial resolution of approximately 2 nanometers or less will be needed to focus on the individual grains. Each member of BayPac works on particle manipulation and analysis using a variety of methods and instruments. To study the isotopic compositions of the dust particles, the Livermore team uses the 200-kiloelectronvolt SuperSTEM, which has a 10- to 100-fold resolution increase over other instruments, and its NanoSIMS (nano secondary-ion mass spectrometry), which is one of only two ion microprobes in the U.S. The team also uses a nuclear microprobe that radiates a sample with 3 megavolts of protons to measure its density.

Scientists in the consortium are studying interplanetary dust particles from Russia's Mir Space Station and the International Space Station to refine the extraction techniques they will use on Wild 2 dust. "These particles are perfect analogs to study," Bradley says, "because they were also collected in aerogel, although at a significantly higher speed (11 kilometers per second) than the Stardust collection speed."

About three years ago, researchers from UC Berkeley's Space Sciences Laboratory developed the "keystone" technique to remove a particle from a sample of aerogel. The term keystone is derived from the tiny wedge that contains the particle track and that is cut out of the aerogel. Detailed optical images of these impact tracks show evidence that particles fragment quite extensively as they project into the aerogel. With the development of the keystone technique, researchers have been able to further refine techniques to remove the fragmented particles. These finegrained particles must be recovered for comprehensive analysis of cometary material.

Livermore researchers are determining which method will best remove micrometer- and submicrometer-size particle fragments from the tracks within keystones. Focused-ion-beam microscopy is one promising method being used to extract 0.1 -micrometer-thick sections of a particle fragment. The thin sections are then examined using the transmission electron microscope, the NanoSIMS ion microprobe, and synchrotron infrared microscopy. Because the focused-ion-beam method destroys most of the particle fragment, only one or two sections can be harvested from each sample. The advantage of this method is that researchers can extract particles as small as 100 nanometers or less from targeted regions and cut them into thin sections. Livermore scientists take samples from specific isotopically anomalous hot spots, that is, areas where the highest 


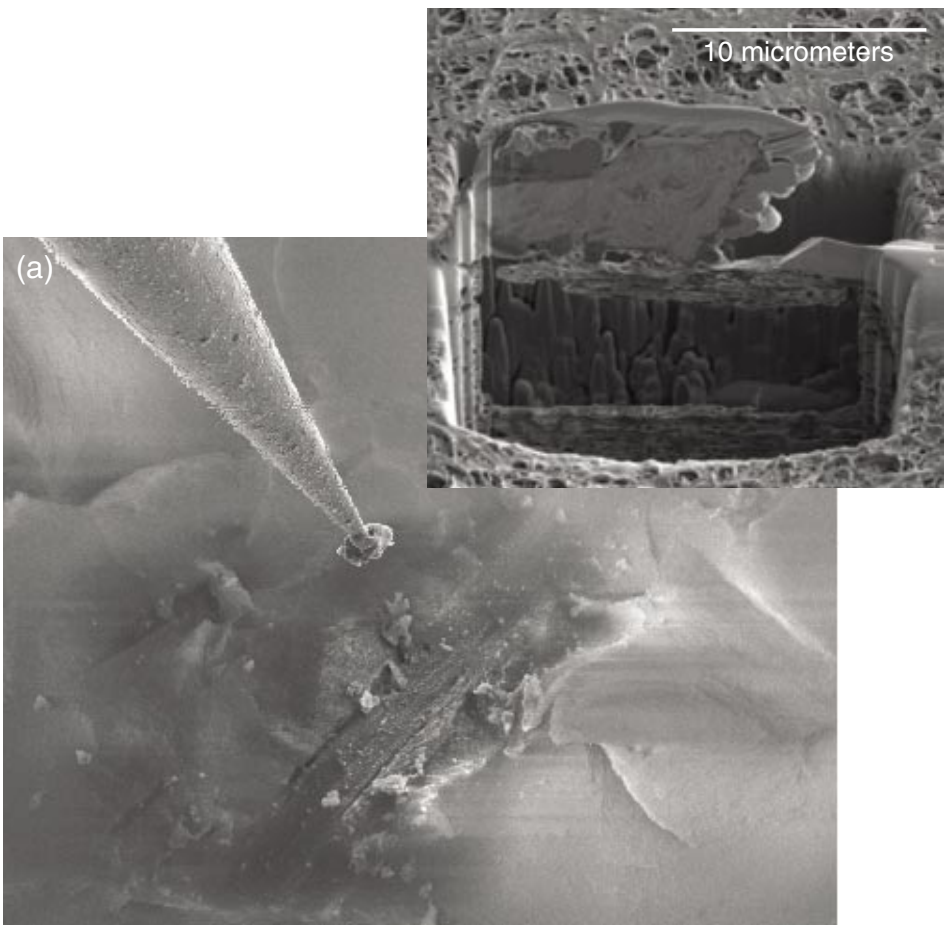

concentration of a given isotope is found. Using this approach, researchers can correlate isotope measurements and mineralogy with nanoscale precision.

\section{Preparing for the Return}

Scientists worldwide will analyze Wild 2's dust, and many of them will travel to Livermore to use the Laboratory's SuperSTEM. Until then, Livermore will continue to refine extraction and specimen preparation techniques. Bradley notes, "For the first time in 30 years, we will be analyzing returned samples, and Livermore will be busy preparing a large quantity of specimens." In addition to collecting particles, Stardust has an optical navigation camera that has captured images of Wild 2's nucleus. Mission planners were surprised when the first pictures relayed back to Earth showed a large, circular nucleus rather than the expected potato shape seen in comets thus far.

In January 2006, Stardust is programmed to eject its reentry capsule, which will parachute to the Utah desert southwest of Salt Lake City. The much-anticipated return of the capsule will perhaps yield more surprises. Scientists are excited about what Wild 2's dust may reveal about the origins of life on Earth.

- Gabriele Rennie

(a) Focused-ion-beam microscopy is used to extract 0.1-micrometerthick sections of an interplanetary dust particle from a sample of aerogel (shown in the inset). (b) The thin sections are then examined using the transmission electron microscope.

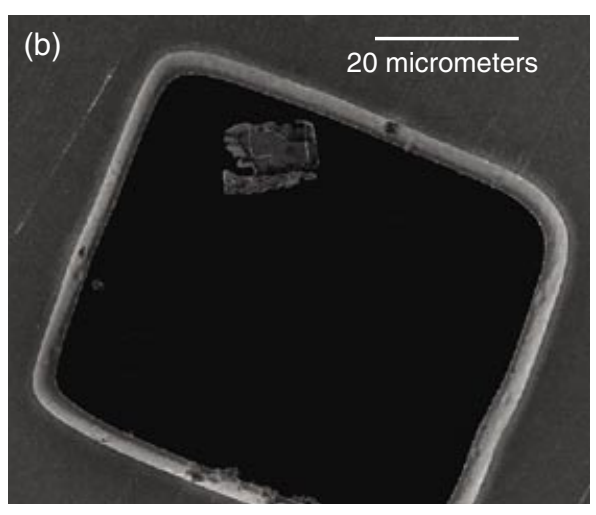

Key Words: Comet Wild 2, focused-ion-beam microscopy, interplanetary dust particles, nano secondary-ion mass spectrometry (nanoSIMS), Stardust, SuperSTEM (scanning transmission electron microscope).

For further information contact John Bradley (925) 423-0666 (bradley33@IInl.gov). 


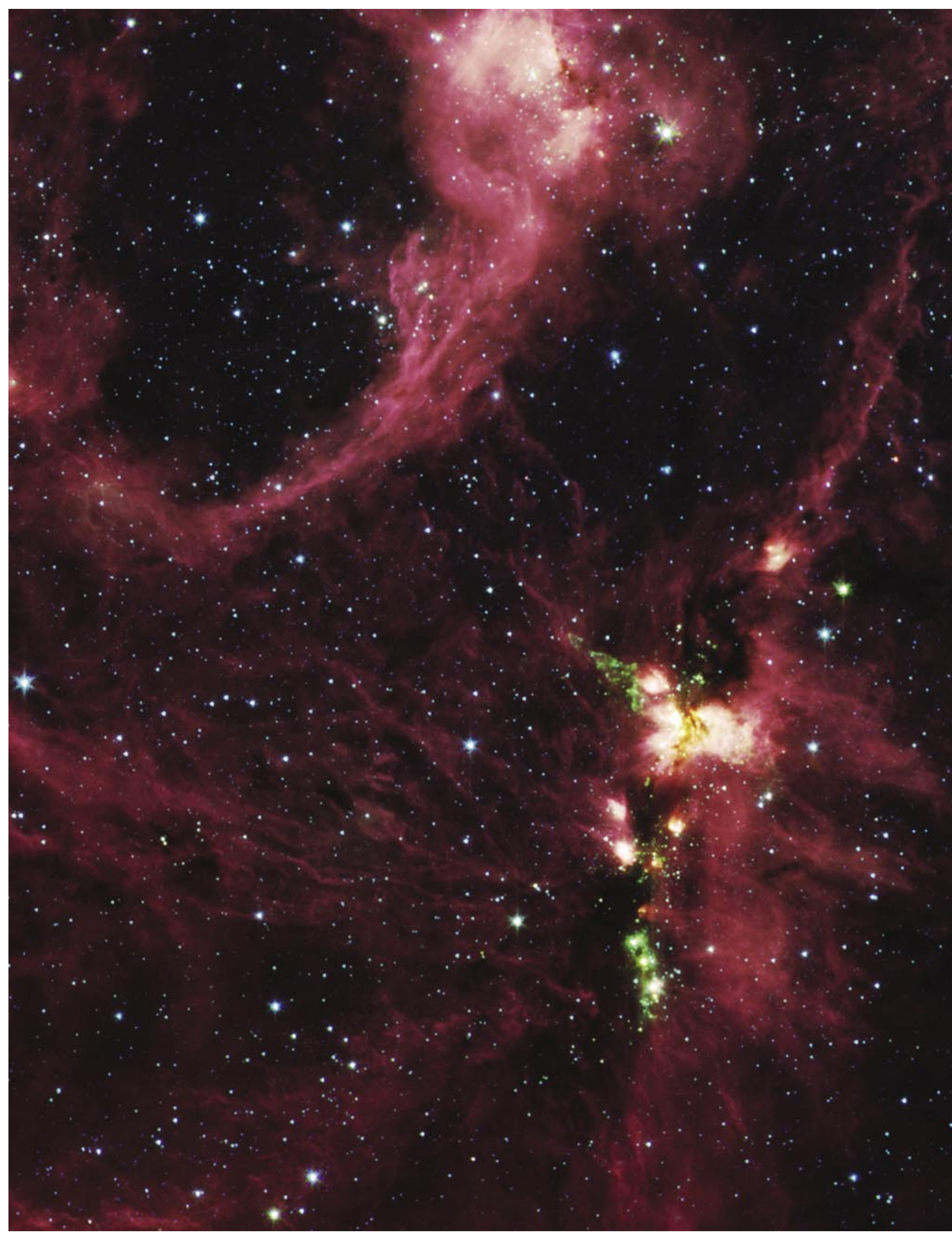

